\title{
Prototyping Mass Nouns from Students' Perspective: A Pedagogical Implication
}

\author{
Md. Saiful Alam \\ Lecturer in English, \\ University of Creative Technology, \\ Chittagong. Bangladesh
}

\begin{abstract}
This paper investigates ESL learners' prototyping mass nouns as a grammatical category. The study is supported by the theory of prototype developed by Rosch in 1970s which plays a wide role in graded categorization of the existent entities in the world. With an expected inspiration from the theory, the prototyping of vocabulary received research attention especially in the pedagogical world. Promisingly, this study seeks to extend the theory to explore a lexico-grammatical category i.e. "mass nouns" from learners' perspective. Actually, the study was directed to find out which prototypical feature ESL students exploit to prioritize some mass nouns as prototypical examples over some other mass nouns, and how far students' experientially and pedagogically perceived "prototypes" of mass nouns help them to correctly grouping up nouns as in mass category. The study was focused on shedding some light on a few pedagogical tips and implications in some likely challenging contexts of teaching mass nouns. The study reveals that ESL students shortlist 'liquids' (such as water, milk, wine, juice etc.) 'gases'(such as hydrogen, oxygen etc.), 'abstract ideas' (such as childhood, anger, safety, knowledge etc.), 'powdered substances' (such as sand, sugar etc.), and some 'natural entities' (such as heat, sunshine etc.) as "prototype of mass nouns" which all are un-individuated and sometimes intangible - meaning uncountable - while the learners recognize 'non-countable' status as the most important prototypical feature of mass nouns. And, the students isolated 'rice', 'wheat', 'hair', 'grass' , 'cotton' and 'coal' as less prototypical mass nouns based on their intrinsic sense that these mass nouns are plural and they even can be individuated. However, the study reflects that the students' perceived prototypes are not sufficient as they selected and considered many of mass nouns as so distant members as countable. It was further found that contextual type shifts of mass-count nouns, arbitrary sematic distributions to lexis, cross-linguistic approach to mass nouns, intrinsic and realistic conception, superordinate-subordinate influence, and perception of enumerating status etc. account for students' this surprise selection of a number of mass nouns as opposite category i.e. count nouns. If pedagogues are non-responsive to these factors and fail to redefine their approaches to mass noun teaching, it is most likely to lead to learners' grammatical inaccuracy resulted from their determiner-number-mass noun mismatch.
\end{abstract}

Keywords: mass nouns; prototype; mass noun prototyping; count nouns; superordinate mass members; nouns

\section{INTRODUCTION}

Grammar of English language is mistakenly or consciously, probably over consciously in many cases, something we as ESL users often exaggeratingly get meticulous about. And, such happens most basically out of our obliviousness about language skills that actually and more importantly need to be pinned down as the main communicative thing about language learning. It's again because of more emphasis, sometimes practically all importance, on language items or language systems (and not apparently on language proficiency) which we commonly term as "grammar" meaning "rules" of language. It is, anyway, "language accuracy" 
(most often thought by both teachers and students to be achieved by explicit grammar teaching) that bothers over conscious, grammatically meticulous language users who put their language in formal communicative contexts in which they always have a fear ( a very bad fear that deters language skills development) of being criticized of their wrong grammatical stuff in their language they produce for communicative purposes. In our context especially here in Bangladesh where... "the teachers and students have always attached great importance to grammar" (Farjana, 2019), and where "English learning" unvaryingly until today in most places means "learning English grammar rules" as much as one can, and where surprisingly a good "English teacher" likewise means a good "grammar teacher", language accuracy is, therefore, very naturally a "prime concern" amongst language learners here. Over and above, in an academic setting especially at university level where language accuracy is one of the large scale considerations, "grammar consciousness" is duly prioritized. But, how far comfortable even our university students are with grammar is still a question. At all events, grammatical accuracy is, for many reasons, out of reach and difficulty with proper categorization of grammar systems and items is what I feel one of the major causes.

Although grammar consciousness or grammar rules of language are something that is naturally instilled in human cognition (Chomsky, 2014), it's in the common cases of second language's grammar learning that explicit grammar teaching and learning doesn't add up that much to guarantee an ESL learner's intended level of grammatical accuracy. Primarily, it is rooted in the cause of pushing out too big load of meta-linguistic stuff (language used to describe language) into students' mind with too poor number of isolated examples of grammatical category. After all, it sounds like a continuous "trial and error" process with grammar learning, which goes on here and in many corners of the world where English is basically an L2.

Actually, at "word-group" level of English syntax, students' consciousness is basically developed, according to Behrens (2008), with "categorization" and "identification", often with grammatical categorization. Precisely, words in the English language are often categorized as "word class" otherwise commonly termed as "part of speech". Eastwood, Heath (1992) specify "...There are eight different kinds of word in English. They are called 'word classes' or 'parts of speech". In fact, grammatical categories are many in the English language such as the most common ones being number, gender, person, adjectives, nouns, pronouns, verbs, adverbs, prepositions, conjunctions, tense, aspects, mood etc. added with so many of their subcategories.

In all this categorization process of the English language at vocabulary level, "prototypicality" is the key used to build up boundary to set exemplars of every category endorsed with "the degree of representativity" (Geeraerts, 2006). Verbs, just as an example, are a category which uses "action" (linguistic term is so far "event") and "state" as the "prototypicality" to mark themselves distinct "word class" from others. However, in terms of "mass-noun" category, there are many members that don't conform to its "prototypicality" (uncountabiity" as commonly perceived), which lead to "mass-noun-number-determiner" confusion and inaccuracy with mass-noun. Even not all adult students can assign some nouns to mass category accurately. From my personal experiences both as a learner and teacher of English, I have seen in many occasions errors often happen with some certain group of mass-noun members such as 'bread', 'soap', 'luggage' and all like them. This study aims at figuring out the possible reasons of students' struggle to handle with the mass-noun members of problematic type. It is really important to take a look at the confusing members of mass noun from students' perspective and check out with the "prototypes" they use to mark out mass nouns in general and how they leave out those problematic members as being the opposite or different category, which altogether will best explain the relationship between prototypical effect and 
mass-noun categorization problem, and influence of other factors involved, if any. It is again essential to find out the fixation and flexibility of prototype in mass noun categorization in wider scale so that students can comfortably deal with extended sense of mass noun members and can use them grammatically correctly.

\section{LITERATURE REVIEW}

\section{Theory of Prototype:}

Developed in 1970s in cognitive sciences, the 'theory of prototype' is one of the well-discussed theories that explain the basis of sense of graded categorization of innumerable entities on earth in accordance with the degree to which they represent the prototypical properties. Different things are given different names to avoid confusion of identicality. For example, we call something as 'bird', something as 'river' and so on. But, what causes each and everything to have different names and identification is recognized as an interesting question in the theory of prototype. Why bird is a bird may be, for example, a curious question. The thing is that when we think of a bird or speak of a bird, we remember a set of common features and patterns by which a living creature is able to be recognized as bird. Prototypical theory argues that the fact is that not all birds have that common set of features sufficiently. Some members of category bird share more features than some other birds. Besides, some features are more central or more regular than others in the category. These central features can be called essential and sufficient attributes that constitute a particular category distinct from other categories. That's to say, on the basis of the absence and presence of some customary features, category members become distinct. For example, let's take girl as a category. Customarily, the most central features of a girl are (i) 'female' and (ii) 'human'. Moreover, one additional feature may be 'adult'. If the first two features are shared by one human being, she may be considered indicative of exemplar of girl category. According to Rosch (1976), a cognitive psychologist and the key person to prototype theory, called the salient / central feature first as 'stimulus' and later termed it 'central member' or 'prototype' of a category. Accordingly, a 'female', who belongs to 'humans', and who is 'adult', may be deemed as 'prototype' or central member of the girl category. Rosch explained what he calls "centrality" of a category in his paper titled "Natural Category" in 1973. In 1976, Rosch et al. went on to explain the notion of the degree of prototypicality or representativity and claimed that it is dependent on the number of features a member seems to have share. Actually, the more features an item shares, the closer it remains to prototype. The fewer features, in contrast, an item characterizes itself by, the more atypical member it accounts for. Accordingly, a robin can be graded as a prototypical member of bird category because it shares all the features of typicality such as feathers, two wings, two legs and the ability to fly while a penguin is a less typical member of the same category on the ground that a penguin lacks some of the typical features of a bird category such as the ability of flight. Thus, according to prototype theory, not every member is equally representative for a category. That's, according to the degree of exhibited typicality, members of a category are recognized with membership at different grade, which is referred to as a 'semantic model graded categorization'. Taking into account of the things categorized in a graded way on the basis of centrality (proximity) and dystality, Galton (1997) explains that a natural category might consist of four like and unlike-prototypical members: (Done )

i. Nucleus: it is the central or prototypical member of the category which has resemblances most in common with the attributes of a category member. "Robin", for instance, would be considered as belonging to nucleus of bird category.

ii. Peripherals: It refers to the various more or less non-central or peripheral members which are recognized as having some but not all of the salient attributives of the central or prototypical members. As mentioned earlier, "Penguin", for example, can have an access to a bird category, however, with a peripheral membership.

iii. Fuzzy edges: 
It refers to such a member that has "the qualities that define a category and the boundaries that limit membership in a category" (Moskowitz, 2005). For example, a "platypus" can be a member locating itself on fuzzy edges because it shares some features of a bird such as beak, tail, etc. but it differs itself from a bird with a unique quality that it lays eggs but distinctively gives milk to its young.

iv. Metaphoricals: It refers to the less or more metaphorical things which are far from the prototype or central members. Interestingly, these sorts of members are sometimes opposite references to the central / prototypical members. Galton gives an example of 'gold leaf' which is a metaphorical member of the prototype of 'leaf' which has some attributes like (1) less or more flat appendages, (2) a short stalk, (3) green in color etc. However, these features are not drawn upon "gold leaf" at all; it's just a metaphorical categorization with no real approximation to "leaf" of trees. We could, therefore, say that it is more metaphorization between things than categorization.

The categorization and gradation explained by the theory of prototype is rather a dealing of innumerable items in category with cognitive approach. Rosch et al. (1976) claim that categorization process depends on 'the real-world attributes' of what is perceived. Actually, categorization in terms of attribute frequency is possible in the perceived world. Therefore, perception mainly affects the categorization. Perception about things around us is created and confirmed in our mind in course of time and through experience. A child's cognitive becomes gradually perceptionally and experientially alive and active about more and more things with the help of adult members of his / her family and society. Human child learns the signifiers and signified system which makes them experienced of the distinction between items and category of things just in this cognitive linguistic way. For example, a child who experiences ducklings playing on his / her yard everyday learns from the older members that it is a category of chicken. Perception in conjunction with experiences is thus connected to humans' conscious identification and categorization of distinctive entities of the world.

According to prototype theory, culture as well influences categorization decision. Although categorization is a common cognitive approach to the world irrespective of cultures, not all cultures give same meanings to a category. The same category may have different cultural significance. Actually, cultural variations lead to the variations of prototypicality, which we might call "category definition and difference" between cultures. For example, some culture may establish 'chair' as a prototypical / central member of furniture category; however the same 'item' may not be accepted as prototypical in some other culture. Rosch (1976) explains that cross-culturally, one particular member may be more privileged in one specific culture than in another. Taken for granted, in Jhenaidah region of Bangladesh, sofa, and cot, and dining table are more prototypical member than chair of furniture category. Geographical diversity, thus, may partly be linked up with the fact of change of centrality of members of a category. In a particular region, for example, where sparrow is not available, robin may be very common type of bird. In such particular geographical region, children might take the features of a robin as the defining characteristics of a bird category and accordingly robin can be a concrete of most central member of bird category to the humans living in that region, which, however, can probably be of less degree of membership elsewhere.

\section{Previous research and knowledge gap unaddressed}

Since its development by Rosch, the theory of prototype with predominant dealing in the knowledge area of cognitive sciences was experimented in philosophy, neuroscience, anthropology, psychology and linguistics. In particular, the prototype theory is already adequately used in the exploration of basically syntactic and lexical categories in the domain of linguistics - lexical semantics and syntax. More into the categorization of word meaning are 
successfully used the prototype effects, as "word meanings are the names of categories, and the meanings of many words display characteristic prototype effects" (Taylor, 2015).

Key figure, Rosch's research paper titled "Cognitive Representation of Semantic Categories" published in 1975 is well said to have opened the curious avenue for studies on lexical and grammatical prototype. There were 200 participants in this research. Rosch investigated how the participants use their cognitive faculty in the categorization of furniture members. The participants were asked to rate some members as good examples of furniture in seven scales. The findings in this research showed that chair and sofa were graded as the central / prototypical member while a telephone was the least prototypical member of furniture category.

Subsequent to Rosch's work, prototypical effects have been investigated in different areas by different people. Especially, prototypical insight has, of late, arrested attention of experts of both general as well as applied linguistics. Precisely, the sense of categorization developed by prototype theory is being strongly considered to be extended to the teaching of categorical components of pedagogical grammar. Zhang (2011), for example, investigated the cognitive approach of categorization of prototype theory in the categorization of English Tenses with an intended recognition of universal cognition of category tense. In this study, three purposes were specifically set to achieve- (i) to investigate the prototype category of tense, (ii) to find out cognitive features of tenses, and (iii) to understand the mechanism of categorization of tense system. The study concludes with arguments that prototype of simple present tense and other categories extend in a chained way of concatenation, ,and expand in the polysemous way of radiation. The study also argues that there is vagueness in the boundary of category tenses, which helps extension and expansion of category of tenses.

Brugman (1981) and Brugman and Lakoff (1988) applied the theory of prototype in their analysis of spatial 'over', which is 'a highly polysemous item' (Leung, 1991) of grammar. Coventry \& Mather (2002) say that they used Rosch's prototype theory as an alternative to 'core sense' approach in explicating the category meanings of 'over'. In their analysis, Brugman and Lakoff proposed a chain of meanings of 'over'. They showed that there is a higher level category of 'over' (which might be called the prototype) which creates a primary sense in the users' minds, but there are some other lower-level category (less prototypical) which create non-primary senses which extend from the higher level category and thus exist as categories in the minds. According to Leung (1991), this study laid some good pedagogical implications in teaching and learning English prepositions.

Zhang (2017) carried out a study to find out the implication of prototype theory in teaching English language vocabulary to Chinese school students. The study suggested that prototype theory has strong utility in vocabulary teaching and learning. It is argued that prototype vocabulary should be taught first because it offers a potential further word-formation which involves lower-level category (less prototypical) of vocabulary learning as well. As to cognitive mechanisms, metaphorical thought in conjunction with metonym should be handled in the learning of vocabulary. Further suggestion was that there should be superordinate and subordinate level vocabulary which should be taught with an ambition of development of inclusive categories (prototypical and less prototypical) of English vocabulary.

Qiang (2014) suggested that prototype theory can be instrumental in the acquisition of category vocabulary. She carried out an analysis which tried to show closeness of prototype theory with theory of markedness, and their suitability in second language acquisition with special reference to category vocabulary of three items- antonyms, gender nouns and 
plysemous vocabulary of English. From the perspective of prototype theory, Qiang analyzed the contrary pairs and came up with findings that contrary pairs i.e. antonyms, pairs of gender nouns, and polysemous vocabulary -all have their markedness. Unmarked word meaning corresponds with the prototype members while those marked correspond with marginal members to a large extent. This, according to the researcher, implies that for ease of and suitability for students' cognitive learning of English vocabulary, at elementary level marked items which are less protypical lying at the edging area of category should be intended to avoid teaching; they should, however, be taught at advanced level. Teaching vocabulary should initiate with unmarked items i.e. prototypical ones.

One study further tracks down an animacy hierarchy of five different types of category nouns which are all previously categorized as inanimate. In descending order, collective nouns, spatial and temporal nouns, concrete nouns, psychological nouns, and abstract nouns are the prototypical members of animacy category. This hierarchy amongst inanimate nouns is expected to result in an impact on word order at syntactic level (Ji \& Liang, 2018).

The previous studies represent that more of 'vocabulary' category appears to have been addressed in good amount, which is obviously a recognized language acquisition concern in applied linguistics, too. Even though not a lot yet, gradually 'grammatical' category has started being investigated including tense category, which might sound less or more a metalanguage aspect. However, no studies on a significant grammatical category i.e. 'mass nouns' from a prototype perspective were found in the existing literature. But, categorization and identification of mass nouns has morpho-grammatical consequences influencing decision of its singular-plural forms, use of determiners with mass nouns at nominal group level (noun phrase construction), subject-verb agreement etc. at syntactical level. The current research is right-expected to help bridge the gap in mass noun categorization from prototypical perspective.

\section{What is noun?}

\section{PROTOTYE OF MASS NOUNS}

Noun is one of the two basic components of a simplest English syntax while the other is verb. That is, at clause level, a noun is the basic component of nominal groups placed in subject or predicate for functioning as subject, object, appositives etc. Basically, the English word "noun" derives from the latin word "nomen" which means "name". A noun is accordingly "a naming word" which identifies and recognizes a thing or a person, or an idea, an action, a quality etc. so as to distinguish from other entities and varieties. Actually everything has a name which is used as a unique identifier making each and every entity as distinct. Consequently, we can define, remember, and separate individual entity to perceive, thus, around us. 'James' ,for example, in James plays good cricket is a noun. Here, we separate James as an individual entity to remember. According to SOKOLOWSKI (2017), noun names an individual, a group or a class. For example, James, (individual), team (group), and peasantry (class) are there nouns. SOKOLOWSKI (2017) also explains that names are categorized into common (man), proper (Delhi), relative (father-son) and non-relative (colleague), positive (honesty) and negative (bribery), connotative (Hitler to mean a killer) and denotative (Hitler, when to mean a name), concrete (iron) and abstract (anger). In the traditional prescriptive grammar, nouns referring to persons or things are mainly divided in two ways: count-noun and mass noun. Naming actually culturally establishes references with some certain features distinguishing an object from others. Prototype theory raises awareness of those features as detective standing in the core of prototyping lexical items. 


\section{What is count noun?}

In the grammatical categorization of the English language at the level of word class, "one way to classify nouns is by categorizing them as count or non-count nouns" (DeCapua, 2010). A count noun, in the simplest sense, refers to the one that can be counted. To add, a count noun can be made into plural and can form a NP with determiners and quantifiers. Hall, (1991) defines a count noun as 'an object of which the individual is a member (e.g., "cat")'. A count noun can be modified, as Gillon (1992) points out, by cardinal numerals (e.g., five,) and quasi cardinal numerals (e.g., several, few, many), while a mass noun by some amount indicators (e.g., much, little). A count noun, too, can be preceded by an indefinite article (e.g., a book). Gillon (1992) also points out that a count noun can morphologically change by adding plural inflections in terms of its number - singular and plural (e.g., book and books), which is never the case with a mass noun. A count noun in generic and indefinite senses becomes plural in form, which doesn't happen with a mass noun. Two examples are in sentence A and B:

A. Books are man's best friend. (Generic sense)

B. I read books. (indefinite sense)

So, the plural morphology, number words' accompaniment, compatibility with determiners which are exclusive to count nouns posit a dichotomy with mass nouns.

\section{What is mass noun?}

In contrast to count nouns, mass nouns refer to a mass of indivisible stuff which can't be counted.

On the web page of Center for Writing Studies of University of Illinois at urban-champion, there is a section titled Grammar Handbook wherein nouns are distinguished as into two- count nouns and mass nouns. ("Grammar Handbook « Writers Workshop: Writer Resources « The Center for Writing Studies, Illinois", 2019). Mass nouns are defined, in the cited page' as 'uncountable' by a number. They can be quantified by some words that signify amounts. Mass noun can be further split into some categorical members as follow:

Liquids: water, milk, butter, oil, blood, coffee, tea, rain, fuel, juice, wine, honey

Powder and grain: rice, sugar, wheat

Natural phenomenon: wood, iron, sand, fire, sunshine, heat, rubbish, salt, diamond, time, gold, silver, diamond, ice, heat, light, steel, coal, weather, grass, garlic, snow

Body parts: hair, meat, flesh

State of being /feelings: childhood, sleep, stress, anger, height, happiness, health, employment, adolescence, satisfaction

Gases: oxygen, air, smoke, hydrogen, nitrogen

Abstract ideas: advice, software, music, beauty, friendship, education, grammar, success, safety, peace, knowledge, logic, poetry, hope, wealth

Games: soccer, tennis, basketball, hockey, football, chess, checker, athletics

Diseases: pneumonia, diabetes, measles, polio, influenza, malaria, hypothyroidism, arthritis

Subjects of study: economics, biology, history, statistics, physics, astronomy, mathematics

Actions: shopping, advertising, listening, swimming, running, anticipating

Materials made of small particles: cotton, cement, sugar, food, paper, mud, chalk, ice cream Names of languages: Spanish, French, English Latin, Sanskrit, Chinese

Superordinate: furniture, soap, work, news, machinery, money, traffic, equipment, mail, cash, bread, baggage, transport, clothing, research, information, advice, homework, machinery, candy, toast, merchandise, sweet, scenery

The most important aspect of a mass noun is that it can't be quantified by a number. It's, therefore, incorrect to say "four woods", "one rice", "three courage" etc. However, by using 
indefinite articles a, an, or a cardinal numeral or semi cardinal numeral before a measurement it is possible to classify or measure a mass noun. Higginbotham (1994) identifies this kind of phrase as "NPs" of mass nouns with count-noun heads. For example:
a. a foot of wood,
b. a bar of soap
c. two pieces of bread
d. several slices of bread

It is important to note that the use of ' $a$ ' and 'a', 'two' and 'several' in the above examples are for count nouns ' foot' and 'bar', 'pieces' and 'slices', obviously not meant or connected directly to mass nouns 'wood' and 'soap' and 'bread'.

Below, there is a test for a mass noun based on some characteristics, which we could extend for consideration as some attributes for prototyping a mass noun:

\section{Tests for Mass Nouns:}

Mass nouns are uncountable.

Mass nouns are quantified by an amount rather than a number.

They have only one form (singular).

They cannot have "a," "an," or "one" before them as modifiers.

They can use "much" (and "little") as a quantifier.

Gillon (1999) presents rather a summary of morpho-syntactic criteria in a contrastive way like the following:

MORPHO-SYNTACTIC CRITERIA:

MASS NOUN

COUNT NOUN

modified by cardinal numerals

modified by quasi-cardinal numerals

modified by indefinite article

modified by "many" and "few"

modified by "much" and "less"

SG /PL contrast

"One" antecedent

\section{Cross-linguistic appraisal of mass nouns:}

However, those above prototypical features of a mass noun might create difficulty determining and marking some nouns, which English language identifies as mass nouns, as being mass class category. 'Luggage' is, for example, can be considered as a superordinate member of mass noun because in common sense luggage is such a thing that people obviously tend to look upon as a countable entity. Let's take an example of an event of your carrying two pieces of luggage in a plane trip away from home, may be to any foreign country. So, when you are checked in for boarding and your luggage goes off you away into the plane's separate carriage, what do you try to remember? Do you remember you have two lagguages (though two luggages is incorrect in prescriptive grammar)? or do you remember you have much luggage? Thus, most likely thing is that the superordinate members of mass noun of the English language fail to apply in cross-linguistic way to learn and understand mass-count noun uniqueness. Takatori \& Schwanenflugel (1992) maintain that superordinate member indicated by mass noun is not universal because it may not be applicable cross-linguistically. For example, 'luggage' being a superordinate member of English mass noun is well-perceived as a count noun in Bangla language. For Moss, Tyler \& Taylor (2007), it's a language-particular outlook of speakers to view lexical items namely 'luggage' as an individuated entity. Therefore, teaching English mass nouns to students with special reference to its superordinate members might sound 
controversial to the learners in terms of their known prototypical features. Takatori \& Schwanenflugel (1992) sensibly get the point that mass-count distinction is something more than the presence or absence of preceding numerals, classifiers and quantificational phrases. This might suggest that EFL and ESL teachers should make sure that cross-linguistic attention effectively involves in appraising and recognizing mass noun categories.

Another factor that can partly have connection to the problemitization of mass-count distinction is language arbitrariness. The meaning attributed to words is sometimes arbitrary and generationally extending. This explains why we hardly account some certain words' meaning as something 'approving' in one language might mean quite 'unacceptable' or even 'offensive' in another . Similarly, noun types arbitrarily might vary from language to language. What is meant to be count noun category in one language may be considered as opposite i.e. mass noun. Casey (1997) termed it as 'arbitrary semantic' distribution to lexis of languages.

\section{Psychological approach to mass noun:}

Research comes up with some explanation and recommendations that mass noun prototyping also includes psychological approach. Especially, why superordinate members such as 'furniture' are included in mass noun can be well explained if it is looked into the issue from a psychological perspective. Casey (1997, cited in http://www.uta.edu/faculty/stvan/stvan98_ch2.pdf ) points out that "count/mass syntax maps not simply to entities in the world, but rather to the cognitive construal of those entities as individuals or unindividuated entities." Markman (1985) explains that superordinate members of mass e.g., furniture, money, jewelry etc. refer to diverse, discrete, countable objects, and they violate the semantic basis of typical mass noun. Mass noun basically refers to relatively mass-like substances such milk, water, sand etc. However, Markman (1985) argues that the superordinate members could also add up to being considered among mass nouns, if their position in hierarchical level is determined and understood obviously. The members which remain at high level of hierarchy should be counted as mass noun while those at a low level should be grouped as count nouns. For example, 'furniture' posits at a relatively high level of hierarchy while 'chair' at a lower level. So, 'furniture' is in just that sense should be included as a mass noun, but not 'chair'. Croft \& Cruse (2004) interpret that "linguistically, names for superordinate categories are often mass nouns when basic level terms are count nouns". Markman reports that investigation on not only English but also 20 more different languages supported this prediction. A study, in particular, confirmed that pre-school children could learn a superordinate member such as 'vehicle' as mass noun in a better way when they were exposed to examples like 'A car is a piece of vehicle' rather than 'A car is a vehicle'. Precisely, superordinate members of mass noun, thus, could be dealt with successfully by the exploitation of psychological functions in the deliberation of language structure and categorization.

\section{Type shifts of mass vs. count nouns:}

Mass-count noun distinction is not independent of some other language and linguistics paradigms. Based on the varying contexts, type of one noun shifts to another (Kang, 1994). Context, for example, is good enough to cause type shift between mass and count nouns. The mass noun 'coffee' in the sentence (a) turns into a count noun in the context that the customer wants two containers of coffee as serving sizes (let's say two cups) for two separate persons.

(a) Two coffees, please. (type shift : mass to count)

Again, in the context of natural kinds of mass noun as a substance, a mass noun changes into a count noun. It is frequent cases with material terms. The sentence (b), for instance, speaks of 
three natural types of 'water' and so it turns into 'waters' with a morphological change of plural ending ' $s$ ', with a count noun shift from its original mass type.

(b) Samples of three waters were collected to put in the lab for arsenic test. (Mass to count) It's important to remember that language is never used without a context. Swarts (2015) puts that context determines meaning. Meaning then helps decide lexical category. Context just as in the sentence (a) very much strongly influences the morpho-semantic shifts between two categories of nouns.

Apart from contextual influence, there are a few other explanations of mass-noun type shift. Pelletier's (1975) 'universal grinder', gives one good ground of how a count noun takes a shift to a mass noun. If an object, which is clearly a count noun, is ground up, it is still referred to by the same name (noun) although it eventually means just a mass of the object as in the sentence (c).

(c) Wash all the apple all over your face. (type shift : count to mass)

\section{Research Questions}

\section{RESEARCH DESIGN}

This research sets out to seek answers to the following specific questions:

i. What "prototypicality" do students use in categorizing and exemplifying mass nouns?

ii. Which are the "prototypical members" of mass nouns singled out by ESL students?

iii. What pedagogical implication does students' dealing with the prototyping of mass nouns have in teaching nouns of mass type?

\section{Significance of the study}

Basically, out of an ESL teaching / learning reality, when it is almost commonly difficult less or more to all ESL users of the English language grammar systems, this study got inspired to investigate the reasons behind learners' struggle with proper categorization and identification of English mass nouns. Practically, as far as it came in my sight and observation, ESL learners quite often make mistakes with mass noun identification, especially when it comes to selecting and using correct determiners with mass noun-headed phrasal expressions as in I don't have "much luggage". The further inaccuracy students go on making with mass-noun usages is the determination and decision of "polarity" (positive / negative) of a mood (declarative, interrogative, exclamatory etc.) influenced by mass noun-determiner-go together norm, such as the grammatical norm that "much + mass noun" usually takes place in affirmative or interrogative mood ( for example: "I have much money" is less acceptable and less normative that "I don't have much money" or "Do you have much money"). As a consequence, ESL learners' language suffers grammatical accuracy partly because of mass nouns, and it puts them on a disadvantageous and often in an embarrassing situation with lesser number of competent ESL learners concurrently involved in all kinds of communicative contexts, more especially and gravely in academic contexts at tertiary levels. This research is expected to come up with some findings and suggestions about how students "categorize" mass nouns with their perceived "prototype", and how much ESL teachers, as accordingly, would probably need to rethink over their "mass-noun teaching strategies" so as to make a significant contribution to the minimization of ESL students' mistakes and lack of confidence with mass noun category decision and related, extended grammar usages. Thus, the present study is believed to simultaneously lay a significant pedagogical implication, as well. 


\section{Data \& and data collection}

By type, this is a rather qualitative research on a grammatical aspect of the English language nouns, added with a small number of occasionally statistical representations. The data comprise sheets of 137 selected mass nouns put by the participants in different table columns in terms of the prototypical proximity level of those example nouns. Actually, the data collection sheet contained a list of 137 individual mass nouns adapted and collected from several authentic English grammar books and put in a jumbled order, and the date sheet involved asking students to categorize and placing the designated nouns from the provided sheet in respected table columns marked as distinct columns for prototypical, less prototypical, and distant members of mass nouns to go in. Students received the mass noun prototyping task sheet (one each) and accomplished the task in the given time on instructions by the simultaneously present researcher. It was a 20 minute-task which almost all the participants were able to finish by the given time. As the researcher was their lecturer, the students appeared all enthusiastic and comfortable in participating in the data sheet task. During the task going on, a few students, however, were found soon struggling with a difficulty grasping the meanings of some certain items of mass nouns in the data sheet. The researcher instantly stepped forward to help them out by reading out the meanings of the pointed mass noun examples both in Bangla and English for the participating students' best convenience. Time came up and during the course when students finished up, the sheets worked out were taken back from the participants which followed sincere thanks from the researcher.

\section{Participants}

Participants of the present research comprise 50 undergraduate students who are all currently doing BA English studeies at the University of Creative Technology Chittagong (UCTC), Bangladesh. The age of the participants ranges between 19 and 24, all having a common background of long 12 years' secondary and higher secondary compulsory academic English learning through grade one to twelve. Moreover, all they have finished, and also currently are taking some major and non-major English language courses at UCTC including Basic Grammar, Grammar in Use, Reading and Writing I and II, Speaking and Listening I and II at the ELL Department of the aforementioned university. Grounded on their previous English learning experiences, the researcher gave a practical thought to himself the targeted participants would be be able to do the task of prototyping mass nouns. The participants were selected randomly as found and gathered at the department after the break of all classes on the data collection afternoon.

\section{Data analysis}

Collected data in the form of mass noun-prototyping sheet filled in by the participants with gathered prototypical features of mass nouns as the participants wrote in the data sheet accompanied by students' prototypical order and classification of mass noun examples put in the three respected sections as per their nearness of prototypicality were, afterwards, analyzed by re-listing and inter-matching all over individual student's categorization and classification of mass noun-membership. Tracking down the frequencies of maximum references of mass noun examples with closeness of prototyicality (used by the students) followed. Then finally came up three inclusive and complete lists of mass nouns put into three distinct groupings such as prototypical, less prototypical and superordinate members, with a set of core features of mass nouns that the students perceive. More findings were tried to be ferreted out from further insight given into the students' right /wrong classification and gradation of 137 mass noun members provided. There was a simultaneous investigation of the potential reasons behind students' mistakes made with their decisions of mass-count distinction, and it was accordingly extended to make some pedagogical implications suggestive of a revision of mass noun teaching strategies. 


\section{FINDINGS \& DISCUSSIONS}

The present study comes up with the following points to discuss and to give insight into, and is led to some conclusive understanding and implications as follow:

\section{Students' perceived mass-noun prototyping features:}

On analyzing the features of mass nouns as perceived and put down in the data sheet by the participants, it was found that the main prototypical aspect of a mass noun, students think, is "uncountablity" which is what universally is perceived. So, all the participants (100\%) uniformly put, first in the listing of mass noun features that a mass noun is "impossible to count". Apart from this main feature (unaccountability), students also associate a few other secondary prototypical aspects with mass nouns, such as (it is) "weighable", (it refers to) "substance" or "quality", (it is) "too tiny in size", (it is) "liquid", (it) "can't be touched, seen" but (it) "can be felt". If summarized, the mass noun prototypical features decided by the students would look like the following simple list:

\section{A mass noun-}

(i) is, first and foremost, “uncountable” meaning it can't be counted. Additionally, a mass noun usually-

(ii) is tiny in size

(iii) refers to qualities

(iv) is liquid

(v) is substance

(vi) can't be seen

(vii) can't be touched

(viii) can be felt

Matching up with the above prototyping features, students singled out and grouped up prototypical, less prototypical and distant mass noun members from the given list of nouns in the data sheet, which actually led the students to miscategorize many mass-noun examples and tracked them as count nouns detailed in the following sections.

\section{Students' perceived prototypical mass nouns}

Students sorted out their perceived prototypical mass nouns from the given list based on the their known prototypical features found in those example nouns. Through analysis, it appeared obvious that the students gathered nouns of five classes of things as the prototypical members three of which are "liquids" such as water, milk, butter, oil, blood, fuel, juice, wine, honey, rain and "gases" such as hydrogen, oxygen, nitrogen, air, smoke, fire, "abstract ideas" such as safety, peace, beauty, happiness, sleep, stress, anger, health, knowledge, hope, friendship, education, success, childhood, adolescence and satisfaction. They actually decided these nouns denoting three types of things as prototypical mass nouns basically because of their quality of being "undifferentiated" or "un-individuated" and therefore "uncountable" which the students in the first place marked as the core prototypical feature of a mass noun. That's, the students determined the core prototypicality of mass noun is "not countable". As the "liquids" and "gases" are not possible to individuate; so it's therefore uncountable meaning mass noun. "Abstract ideas" are "intangible" which means they can't be counted as something to be counted has to be seen first. "Intangibility" is an additional feature of mass noun pointed by students. The fourth type is "natural phenomenon" such as ice, sand, sunshine, mud which students considered as prototypical mass nouns as they have core prototypical features. Powder is the fifth type of prototypical mass nouns exemplified by salt, sugar, sand, cement etc. 


\section{Students' wrongly perceived less prototypical mass noun-members}

According to students' perceived prototypicality for mass nouns, $76.44 \%$ of them sorted out 'rice', 'wheat', 'hair', 'grass' , 'cotton' and 'coal' as being less prototypical mass noun-members. Despite these referred nouns belonging to "a class of objects that we can talk about only collectively as an undifferentiated whole group" (Lester, 2013), students tend to believe that they can even count these objects and can split them into smaller individuals, which gives students an impression of plural idea and countability status attached to these nouns. One most possible reason behind students' considering these set of nouns to be less prototypical is that students feel possibly these nouns are rather close to countable as they seemingly hold a false belief that "these nouns are intrinsically plural in nature, but singular in grammatical construction" (Bennett, 2005). But, realistically, none of us count them on any occasion. So, ESL teachers, in such a case of students in dilemma, are suggested to break students' wrong perception by explaining that we don't realistically have to separate the individuals of these nouns in question, and we use them as undifferentiated mass meaning they are without doubt mass nouns and no wrong calling them prototypical ones.

\section{Students' wrong categorization of 'coffee' and 'tea':}

The study shows that a remarkable number of students (63\%) marked "coffee" and "tea" as the nouns of "countable" category even though their general grammatical category is uncountable. This decision, as found out by Kang (1994), may be made by abiding by a contextual guarantee in which type shift of a mass noun to a count noun takes place. In our everyday tea-coffee context, we always exchange our discourse in a set meaning with "tea" and "coffee". Actually, we demand and decide "a cup of tea" or "a cup of coffee", which is dealt as countable items, always especially in a tea or coffee shop as usual ordered / requested just like two items of drink. Even, it is further likely that participants' cross-linguistic background has a connection with tea and coffee being perceived as count nouns. The participants, for example, in this study are all Bangla speaking ESL learners. It is in the Bangla language that its speakers deal with coffee or tea as a countable item both at home and outside in tea stalls, coffee houses and elsewhere in all tea /coffee contexts. In Bangla, tea is ordered in a tea shop or elsewhere tea is dealt in by saying: Amakle (me) ekta (a) chaa (tea) diyen (Give) (i.e. in normal English syntax the order for tea is commonly "Give me a tea, please".) This "tea-coffee" case of the Bangla speaking students so far implies that English language teachers of Bangla speaking ESL students should be mindful of offering their learners some cross-linguistic insight when putting forward grammar of mass-noun. More importantly, grammatical category especially mass nouns should be taught, keeping in mind the options of those nouns' likely "type shifting", just as happens in the "tea-coffee" case, with varying contexts because language, according to Swarts (2015), is never used without context. This, otherwise, strengthens the opinion that the teaching of language items i.e. grammar should essentially needs a shift to the "context-based" teaching (formally called "grammar in use") from the age-old explicit grammar practice with isolated examples (often out of context and without co-texts).

\section{Students' wrong category perception of languages and diseases, games, and subjects of studies}

The study suggest that superordinate categorization, as argued by some researchers, may have played an influencing role in 91\% participants' grouping different individual "languages", individual "diseases", and individual "games" as count nouns although they are actually have all have the grammatical category of mass nouns set out in prescriptive grammar. All the individual languages such as Spanish, French, English, Latin, Sanskrit, Chinese, and all the individual diseases such as pneumonia, diabetes, measles, polio, influenza, malaria, hypothyroidism, arthritis are reported by the participants as count-nouns, which is wrong in standard English grammar. That's, students find them all so distant from mass noun 
prototypical features that they attach just the opposite category to them being countable even though their actual category is mass. Superordinate members and their hierarchical members with lower levels of members in line with mass-count noun distinction system, as suggested by Markman (1985), Croft \& Cruse (2004), seemed to have worked. As Markman (1985), Croft \& Cruse (2004) maintain that "language" is a superordinate member which remains at higher level while Spanish, Latin, English etc. are lower level of its individual members which should, as per the system, be called countable nouns. Participants just did it; however, it is not the case. It is similar, too, to assume that category decision for "disease" as a higher level of superordinate member i.e. mass noun while pneumonia, diabetes, measles, polio, influenza, malaria, hypothyroidism, arthritis as some individual members of lower level have been plainly decided to fit in count nouns, which is again apparently not the real case because prescriptive grammar clearly classifies them as mass nouns. Correspondingly, individual members of general term "game" such as soccer, tennis, basketball, hockey, football, chess, checker, athletics etc. are gathered in count noun category but they are actually included in mass noun category in traditional grammar books. Finally, it is found that students' same mindset accounts for the individual branches of knowledge such as economics, biology, history, statistics, physics, astronomy, mathematics etc. being considered in count noun category, which is just opposite to their real grammatical category i.e. mass noun . To sum up, superordinate- subordinate categorization system has just opposite effect on students when it comes to categorizing individual diseases, games, languages, and knowledge branches. Actually, students tended to plainly categorize the mass nouns, which are distinct individuals of a higher level of general member, as count nouns. So, students' mass noun decision on the individual members of language, disease, games, and branches of knowledge can be coordinated by offering them just one mass noun test with indefinite article. For example, "Hasan is a footballer and he plays a football / football". In such contextual syntactic structure, students' category decision might immediately switch to mass noun which they primarily tended to argue as count nouns. Students' general grammar consciousness can, thus by determiner- mass noun test, facilitate opting to decide mass noun category of a number of individual members of a few general umbrella terms namely "language", "disease", "game", and "knowledge".

\section{Students' further incorrect categorization of superordinate members}

This study gathers evidence that all the participants cite some superordinate members in question as count nouns which are actually gathered in mass noun category in grammar. Just like a common prototype perception about mass noun in students prompts them on category decision of "furniture", "transport", "food", "time", "height", "equipment", "machinery", "traffic", "grammar", "money", "music", "poetry", "cash", "wealth", "advertising”, "mail”, "clothing" etc. as "count nouns". That is, it was an obvious way with the students that almost all of them regard the aforementioned set of mass nouns as some odd members that go straightly "distant" from mass noun prototype. Students perhaps approach this set of superordinate members of mass noun with a psychological mindset. It accounts that they always refer to the lower class of members by the superordinates. For instance, students' immediate attention is up to "chairs, "tables", "sofa" etc. when they make reference to "furniture". As chair, table, sofa etc. are treated as some discreet countable objects, and as their general term is furniture, they, therefore, follow their psychologically developed "reference system" and use this psycoexperiential clue to sorting out "furniture" as a count noun. Likely, the same clue can be sourced behind the cases with the rest of aforementioned superordinate members being reported as count nouns by students. "Time" is, for example, a superordinate member of "minutes", "seconds", "days, "weeks", "months", and "years" etc. which all fall into the count noun class. Therefore, students are possible to take "time" in count noun groups and contrast with mass noun. Thus, "transport" is psychologically shifted into count class as students mean 
a few count nouns such as "buses", "cars", "trains" etc. when they think of "transport". Likewise, students source countable things such as "hamburgers", "apples" etc. when they think of "food" and continue to similarly mean "meters", "centimeters", etc. by "height"; "cameras", "sound recorders", "bats", "computers" etc. by "equipment"; "livers", "pulleys", "screws" etc. by "machinery"; "cars", "buses", "trucks", "rickshaws", "taxies" etc. by "traffic"; "prescriptive rules" (that deal with right / wrong) by "grammar"; "currencies" such as Bangladeshi Taka notes by "money"; "songs" by "music"; "poems" by "poetry"; "currency" notes by "cash"; "houses", "cars", "plots", "flats", "deposits" etc. by "wealth"; items shown and described on television, newspapers by "advertising"; "messages", "letters" etc. by "mail"; "shirts", "pants" etc. by "clothing". More precisely, students underpin their categorizing all the mentioned superordinate members as count nouns instead of mass nouns by their being psychologically tapped by the lower level countable members of those higher level of superordinate nouns. This is what Casey (1997) links to students' cognitive construal of the entities as they have grown up with a development of psychological mindset about the superordinate members of mass nouns and classify them as some "diverse, discrete, countable objects" (Markman, 1985). With such a grammatically incorrect behavior of students towards a big set of mass nouns, teachers might adopt some means to put the leaners out of errors by uncovering to them with a simple key that in the hierarchy technically the superordinate members ( such as "furnitutre") are mass nouns and those ranked lower (such as, sofa, chair, table) are count nouns. A second way out may be the teachers might make some changes in the syntactical elements when they present sentence examples with superordinate members as in "A car is a piece of vehicle" rather than "A car is a vehicle". This type of syntactical alteration has been reported instrumental in teaching superordinate members as mass nouns.

\section{Students' misconception about measured nouns}

Participants almost commonly (89\%) identified some mass nouns as count nouns, which is obvious grammatical errors with noun-class categorization. 'Wood', 'gold', 'silver', 'diamond' 'steel' 'paper' 'chalk' 'ice-cream' 'soap', 'bread' 'baggage' 'candy' 'toast', 'iron' are all categorized as mass nouns in prescriptive grammar of the English language but the participants sorted out them all as opposite category i.e., count nouns. Students in their real life situations deal with this set of mass nouns with a practical mindset of "measurements", "sizes", and "shapes" as some items of objects that we hold in hands, pack in, etc. always with a counting necessarily. Some measuring units that go with the nouns above include piece, bar, slice, loaf etc. which are themselves count noun category. For example, "a piece of candy", a "bar of soap", a "slice of bread", and the like are commonly found as expressions in the grammar of standard English. So, the sense made out measuring unit and mass noun-go together fashion leads students to mistakenly refer to these special set of nouns with an extended reference to their "countable measurement units" and, thus, eventually construe their category as the count ones to be individual items. It implies that mass-count categorization is not "universally" alike (Takatori \& Schwanenflugel, 1992). Cross-linguistically, Bangla language even doesn't use these measurement words; it directly uses numerals before these nouns. For example, Bangla native speakers say "two chocolate(s)", "three bread(s)". This conceptual representation of countability in their own language may have influenced the participants to approach this particular set of nouns as count category instead of mass ones, which Moss et al (2007) explains as "language specific" outlook on lexical items as individuated entities. Students' mistakes with this set of mass nouns categorized as count nouns may also be partly linked to the universal "arbitrary" aspect of "semantic distribution" (Casey, 2007) to lexis. Therefore, it is imperative that ESL teachers should be personally more attentive to the appraisal of arbitrary aspects of languages and L1 influence, and make their students similarly aware of the bi-lingual and cross-linguistic approach to this set of special mass nouns so that they can make sense of especial cases beyond their simple prototyping practice of mass nouns. 
Students further extended their psychological perception and cross-linguistic notions as they identified 'employment' 'work' 'information', 'research' 'homework' 'scenery', 'advice', 'shopping', 'news' and 'logic' as count nouns, not mass ones. These nouns basically have a real pattern of use with an aspect of being "enumerable by numeric piece in contrast to other English abstract mass nouns like patience" (Santos, 1990), which might have mainly accounted for the participants' marking out the above mass nouns as count ones. Participating students all along use 'one news, two news' etc. in their Bangla speaking, which might be partly operating as a factor for their count noun decision of "news" which is indeed a mass noun. Polzenhagen (2007) and Ning (1998) point out that theory of prototype also asserts that, in cognitive-linguistic approach, category variation and classification with meaning differences of the same category is also subject to variations across linguistic, social, religious, and political culture and environments. So, teachers in such a context ought to shift the 'enumerating' status of the above nouns in Bangla to their 'abstract' identity in English for convenience. After all, teachers should remind the learners of the fact that "a mass noun is not homogeneous in any strict, literal sense" (Weseliński, 1998), and ,therefore, the "protoypes" they use as the standard to make mass-noun decisions as a grammatical category doesn't essentially and equally function and apply to all mass noun members of the English language. So, other interfering factors are supposed to get an unavoidable attention in the pedagogy of grammar categories especially more troublesome and misleading ones such as mass noun category.

\section{PEDAGOGICAL IMPLICATIONS}

Based on the worrying findings on students' huge erroneous identification and categorization of mass nouns, the study lays the following related pedagogical implications for all concerned with ESL teaching with occasions of grammar category teaching at morpho-systactical level involving meaning distributions in line with contextual language uses:

A. This study suggests that teaching of mass nouns should get started with students' prototypical examples because students will get easy and immediate unfailing grasp of these most matching examples, which they may be ready to gradually extend to the closer members and distant exemplars. If the confusing examples are tried in the first place to make them perceive as mass nouns, it might create a disbelief and difficulty generated from their perceived mass noun prototypes and they are likely to end up with a very bad impression about the teacher and may immediately shut down their learning curiosity at least at the moment about mass nouns in details. Categorically, this study suggests that prototypical examples of "liquids", "abstract ideas", and "gases" be first taught when in a lesson the focus is on mass noun. It is because "...category membership of prototypical examples of objects should be learned before that of less good examples" (Johnson-Laird, \& Wason,1977). It is otherwise suggestive of the theoretical fact that prototypical examples are the basic level of members and are good representatives of a category. Schmitt (2000) believes that "the most prototypical examples be chosen as first examples when exemplifying a category."

B. When there is already a good practice of most prototypical examples of mass nouns i.e. "liquids", "abstract ideas", and "gases" with an obvious quick production and recognition of mass nouns with fewer errors at a later stage, students should only then be directed to less prototypical examples. At this stage of move-to-less prototypical, teachers might want to add more attributes to a mass noun in addition to learners' own perceived limited mass noun prototypical features. As a consequence, students get ready to expand their outlook and create an inclusive perception to enlist many new examples in mass noun category.

C. To deal with the "type shifting" principle, teachers should consider teaching mass vs. count nouns in line with occasional context shifts. ESL teachers should be teaching especial mass-noun examples like "tea" and "coffee", which Riemer (2010) thinks have 
"shift flexibility", not as isolated examples in explicit grammar teaching fashion that's been coming through ages hand in hand with Grammar Translation Method (GTM). It's duly emphasized that flexibility of techniques maximize language understanding and learning. So, special mass noun items with high "shift flexibility" and contextual meaning variation possibility such as "tea" and "coffee" should be taught by putting them as words and nominal groups (noun phrases) in practical language use at syntax and clause level and create clear sense of how their shift changes turn their category from mass to count and vice versa.

D. In the cases of "language" "disease", "games" and "subjects" of studies, it is found that just the opposite of "superoridate- lower membership" categorization type occurs in the students' ideas and perception. That is, there are a good number of superordinates which are non-count. Furniture is, for example, a superordinate and therefore noncount but lower members such as chair, table, sofa etc. are count nouns. In line with that understanding, students identified the lower members such as Spanish, German, Chinese etc. of the superordinate "language" as count nouns. However, Spanish, English, German and so forth are all are said to be non-count in grammar books. Students also made similar mistakes by identifying lower members of games such as football, soccer, hockey etc. as count nouns. Their mistake repeats in the categorization of branches of knowledge such as 'economics, history, physics etc. Therefore, it is a good idea that teachers should not "refer to one or more entities as individually belonging to a mass superordinate category" (Pelletier, 2009). Precisely, teachers should be dealing with the refereed nouns as the matching and unmatching membership between 'superordinatesubordinate' categories, as in this case the system doesn't work the way correctly as in some other special nouns such as "furniture", "traffic" etc.

E. A big number of subordinate members of some superordinates such as furniture, machinery, grammar and so on are identified as count nouns, which is wrong. To help students out, teachers might make some changes in the syntactical elements when they present sentence examples with superordinate members (should be put in a phrasal structure) as in "A car is a piece of vehicle" rather than "A car is a vehicle". This might lead through a practice to students' habit of perceiving a measuring / numerating word plus a count non following a superordinate, with a gradually developed, conscious feeling that the superordinates are non-count i.e. mass nouns. Previous research also has reported that such syntactical alteration has been instrumental in teaching superordinate members as mass nouns. (Done)

F. Language teachers must emphasize on and give thought to themselves about "the fact that language is arbitrary"(Gardner-Bonneau \& Blanchard (Eds.). 2007), which implies that every two languages may not find one to one correspondence with the single type of grammatical category such as mass nouns. More specifically, Bangla speaking ESL learners might get confused with English mass nouns such as those as mentioned in 4.7, by the existence of "numericals plus mass noun" in, let's say, Bangla. Again, teachers should shift the "enumerating" status of Bangla nouns to "abstract" of English mass nouns, which might help students remember a specific "status change" of a particular group of tricky mass nouns of English language with a blocked understanding created by Bangla.

\section{CONCLUSION}

Prototyping and graded categorization has been going hand in hand since time immemorial when first humans began to assign meanings and category names (signifiers) to distinct one thing (signified) from the other (signified). For detecting, selecting, and separating one entity from so many others as a distinct individual "existent", humans has ever used "prototypes" and laid boundaries for other categories of entities to mark themselves distinct across those 
boundaries. This is even now evident and believed that "prototyping" is equally used for grammatical categorization with language items. In this study, a particular item of lexicogrammar i.e. "mass nouns" and prototypes used by ESL students are investigated.

Explicit grammar word-class (e.g. nouns) with their sub categories (e.g. "mass nouns") are currently being investigated with the application of theory of prototypes. This study also applies Rosch's theory of prototype to find out what prototype ESL students use to categorize mass nouns. Practically, many students, as far as I have seen, make mistakes with a set of massnoun members and put wrong determiners in phrasal constructions, and occasionally make them into plural forms which is not typically the case with a mass noun because usually a mass noun doesn't turn into plural form. So, I have often heard students say (and it is even no surprising to hear an immigration officer at a non-native English speaking country's airport) "how many luggagues do you have with you?" This sentence is, according to what prescriptive grammars detects, wrong because "luggage" is categorized as a mass noun which is not supposed to be followed by a numerical determiner such as "many", and it is again not normally supposed to take itself into 'plural' form. Evidenced with many similar instances, it is, therefore, significant to find out students' understanding about mass noun category and the "prototype" which they have built up from grammar study as well as teachers' explanations, and which they use in identifying mass noun as a sub category of nouns. Additionally, the study was inspired to lay some pedagogical implications as for mass noun teaching to ESL students especially by offering some ways and means of how to deal with a confusing set of mass nouns.

Collected from some authentic grammar books, a list of jumbled items of mass nouns was made and distributed amongst a group of 40 undergraduate students of English major studies to work out and come up with those nouns from the table labeled as prototypical, less prototypical, and distant members of mass nouns. Before that, the participants were first asked for writing out "prototypical' features of a mass noun they know and believe in the designated data sheet to write in.

Taken back from students after they were all done with their tasks on the data sheet, prototypical features of mass nouns as the participants wrote in the data sheet, and also students' categorization of mass noun examples put in the three sections as per their nearness of prototypicality were, afterwards, analyzed in terms of listing and matching all over individual students' sheets. Through the analysis, the study ends up with the following findings:

As found in the analysis of this study, the students use "uncountability" as the most prototypical aspect of mass nouns. Students also attribute roughly seven secondary aspects to mass nouns which are (i) "weighable", (ii) some "substance" or "quality", (iii) "tiny" in size, (iv) "liquid", (v) "can't be" touched, (vi) "can't be" seen (vii) "can be" felt. However, checking out with these prototypical features in the given list of nouns (which are all mass nouns indeed), the students happened to mistake many mass noun examples for count nouns, which suggests that students' used and perceived "prototypical" features don't really suffice to deal with massnoun categorization of the English language in large scale. In addition, there are apparently some external factors which must be taken into account in dealing with English mass nouns both from language leaners' and teachers' perspectives. These factors are put below, following the prototypical members of mass nouns:

The study shows that almost all the students find most of their known "prototypical mass noun features" in five particular things which are (i) "liquids" such as water, milk, butter, oil, blood, fuel, juice, wine, honey, rain, (ii) "gases" such as hydrogen, oxygen, nitrogen, air, smoke, fire, (iii) "abstract ideas" such as safety, peace, beauty, happiness, sleep, stress, anger, health, knowledge, hope, friendship, education, success, childhood, adolescence and satisfaction, (iv) 
"natural phenomenon" such as ice, sand, sunshine, mud, and (v) "powder" such as salt, sugar, sand, cement.

The study also shows that 76.44\% students approach 'rice', 'wheat', 'hair', 'grass' , 'cotton' and 'coal' with less or more confusion and mark them as less prototypical members of mass nouns. They feel it is still possible to count these items of things and can further be split into individuals, which partly leads students to perceive them as count nouns; not any accident if they do. One of the reasons behind students' considering them as possibly countable is that they believe these nouns are intrinsically plural in nature, but singular in grammatical construction. But, realistically, we don't count them at all with tiny constituting individuals split off.

It is found further in the study that $63 \%$ students identified "tea" and "coffee" as count nouns even though their general grammatical category is uncountable. This happens because of type shift of mass noun to count noun in varying contexts in which "tea" and "coffee" change into meaning of containers i.e. a cup of tea or a cup of coffee instead of liquid i.e. "drink" itself, which practically students and everyone order for or ask for with a count or number. It happens also because of Bangla and English cross-linguistic influence upon students' perception.

The study comes up with another interesting finding that students make wrong use of 'superordinate and subordinate members' category distinction system in the cases of the categorization of diseases (pneumonia, diabetes, measles, polio, influenza, malaria, hypothyroidism, arthritis), games (soccer, tennis, basketball, hockey, football, chess, checker, athletics), languages (Spanish, French, English, Latin, Sanskrit, Chinese), and subjects (economics, biology, history, statistics, physics, astronomy, mathematics) . As per the system, language is, for example, a superordinate, which is supposed to be a mass noun, and it has lower -level of individual members like Spanish, English, German and so on which are supposed to be count nouns. Students do so but it is really the opposite case because above mentioned lower members of languages, games, diseases, subjects are all deemed to be mass nouns as a noun category. So, this is an obvious instance of insufficiency of students' prototype perception regarding mass noun categorization, which opens chances for students making mistakes with these good number of mass noun members in accompaniment with occasional wrong selection of determiners and plural numbers.

Superordinate members namely furniture, transport, food, time, height, equipment, machinery, traffic, grammar, money, music, poetry, cash, wealth, advertising, mail, clothing etc. are commonly mistaken for count nouns by the students; however these nouns are grouped in mass-noun category in formal grammar of English. There is a psychological and cognitive mechanism in students which explains why. Actually, students tend to mean lower class members by superordinates such as chairs, tables, sofa by furniture. As chairs, tables, sofas are treated to be some discreet countable objects, students consider their superordinate member 'furniture' also to be count noun being just opposite to their perceived mass noun prototype. Students on the same ground go to classify transport, food, time, height, equipment, machinery, traffic, grammar, money, music, poetry, cash, wealth, advertising, mail, clothing all as count nouns, which is again their wrong categorization.

Students don't find mass-noun prototypicality in measured nouns such as 'wood', 'gold', 'silver', 'diamond', 'steel', 'paper', 'chalk', 'ice-cream', 'soap', 'bread' 'baggage', 'candy', 'toast', 'iron' etc. ; so they categorize this set of nouns as opposite i.e. count nouns although they are actually placed in mass noun category in English grammar. It happens because in real life situations, students deal with this set of mass nouns with a mindset of measurements, sizes, and shapes as 
items of objects that we hold in hands, pack in, etc. always with a counting. Some measuring units include such as piece, bar, slice, loaf etc. For example, "a piece of candy", a bar of soap, a slice of bread and the like. So, every time students refer to these special set of nouns, they actually refer forward to their countable measurements and, thus, construe these nouns ultimately as count nouns meaning individual items. Besides, it is also because crosslinguistically, Bangla language even doesn't use these measurement unit words like a piece of, two pieces of etc. ; it directly uses numerals before these nouns. For example, Bangla native speakers say "two chocolate(s)", "three bread(s)" and so on, which dismisses the "universality" about mass-count categorization irrespective of languages.

Last but not the least, students also extend their psychological perception and cross-linguistic notions and they classify 'employment' 'work' 'information', 'research' 'homework' 'scenery', 'advice', 'shopping', 'news' and 'logic' as count nouns, not mass ones. These nouns basically have a real pattern of use with an aspect of being enumerable by numeric piece in contrast to other English abstract English mass nouns like patience, which accounts for why students mark out the above mass nouns as count ones. Practically, the participating students all along use 'one news, two news' etc. in their Bangla speaking, which is partly operating as a crosslinguistic factor for count-noun decision about the refereed items of nouns.

This study sums up that the mass noun "prototype" that ESL learners construe and perceive isn't practically close to working for perfectly identifying and correctly categorizing a good number of nouns which actually belong to mass noun family. This weak connection between prototypes and other distant members of mass nouns leads to students' ending up with mass noun-number-determiner errors in the English language while they produce in writing or speaking it. As found, the involved other factors which influence students' mass-count decision essentially need to be taken into good account and mass-noun pedagogical decisions have to be, at the same time, adjusted in line with the implications tracked down in section 5 in this study.

Acknowledgement: The researcher feels endlessly grateful to his superb Nottingham University professor Dr. Derek from whom he learned word groups and other grammar categories and their functions, which has been so much of help for the researcher.

\section{References:}

Alam, M.S.(2020)

Behrens, H. (Ed.). (2008). Corpora in language acquisition research: History, methods, perspectives (Vol. 6). John Benjamins Publishing.

Bennett, T. (2005). American English for Filipinos. Pasig City, Philippines: Anvil Publishing Co.

Bobb, S., Kroll, J., \& Jackson, C. (2015). Lexical constraints in second language learning: Evidence on grammatical gender in German. Bilingualism: Language and Cognition, 18(3), 502-523. 10.1017/S1366728914000534. (Questionaire adopted from this research)

Brugman, C. (1981). The story of 'over'. M.A. Thesis, University of California at Berkeley. Reprinted by the Indiana University Linguistics Club.

Brugman, C. (1988). The story of 'over': Polysemy, semantics and the structure of the lexicon. Garland Press.

Brugman, C., \& Lakoff, G. (1988). Cognitive topology and lexical networks. In G. W. Cottrell, S. Small, \& M. K. Tannenhaus (Eds.), Lexical ambiguity resolution: perspectives from psycholinguistics, neuropsychology and artificial intelligence. San Mateo, CA: Morgan Kaufman Publishers.

Chomsky, N. (2014). Aspects of the Theory of Syntax (Vol. 11). MIT press.

Coventry, K. R., \& Mather, G. (2002). The Real Story of Over?. In Spatial Language (pp. 165-184). Springer,

Dordrecht. 
Croft, W., \& Cruse, D. A. (2004). Cognitive linguistics. Cambridge University Press.

DeCapua, A. (2010). Grammar for teachers. New York, NY: Springer.

Eastwood, J., \& Heath, M. (1992). Oxford practice grammar. Oxford: Oxford University Press.

Farjana, N. (2019). [online] Dspace.bracu.ac.bd. Available at:

http://dspace.bracu.ac.bd/xmlui/bitstream/handle/10361/3315/10103014.pdf?sequence=1\&isAllowed=y [Accessed 30 Oct. 2019].

Galton, A. (1995). Verb aspect and prototype theory. In Proceedings of the European Conference on Cognitive Science (ECCS'95) (pp. 121-128).

Gardner-Bonneau, D., \& Blanchard, H. E. (Eds.). (2007). Human factors and voice interactive systems. Springer Science \& Business Media.

Grammar Handbook « Writers Workshop: Writer Resources « The Center for Writing Studies, Illinois. (2019). Retrieved from http://www.cws.illinois.edu/workshop/writers/massnouns/

Geeraerts, D. (2006). Prototype theory. Cognitive linguistics: Basic readings, 34, 141-165.

Gillon, B. (1992). Towards a Common Semantics for English Count and Mass Nouns. Linguistics and Philosophy, 15(6), 597-639.

Gillon B.S. (1999) The Lexical Semantics of English Count and Mass Nouns. In: Viegas E. (eds) Breadth and Depth of Semantic Lexicons. Text, Speech and Language Technology, vol 10. Springer, Dordrecht

Hall, D. (1991). Acquiring Proper Nouns for Familiar and Unfamiliar Animate Objects: Two-Year-Olds' WordLearning Biases. Child Development, 62(5), 1142-1154. doi:10.2307/1131158

Higginbotham, J. (1994). Mass and Count Quantifiers. Linguistics and Philosophy, 17(5), 447-480.

Ji, J., \& Liang, M. (2018). An animacy hierarchy within inanimate nouns: English corpus evidence from a prototypical perspective. Lingua, 205, 71-89.

Johnson-Laird, P. N., \& Wason, P. C. (Eds.). (1977). Thinking: Readings in cognitive science. CUP Archive.

Kang, B. (1994). Plurality and Other Semantic Aspects of Common Nouns in Korean. Journal of East Asian Linguistics, 3(1), 1-24.

Lester, M. (2013). English articles and determiners up close.

Leung, M. W. K. (1991). Prototype theory and teaching English prepositions. Perspectives (City University of Hong Kong), 3, 89-97.

Markman, E. M. (1985). Why superordinate category terms can be mass nouns. Cognition, 19(1), 31-53.

Moskowitz, G. (2005). Social cognition. New York, NY: Guilford Press.

Moss, H. E., Tyler, L. K., \& Taylor, K. I. (2007). Conceptual structure. The Oxford handbook of psycholinguistics, 217-234.

Murphy, G. (2004). The big book of concepts. MIT press.

Ning, Y. (1998). The contemporary theory of metaphor: a perspective from Chinese.

Pelletier F.J. (1975) Non-Singular Reference: Some Preliminaries. In: Pelletier F.J. (eds) Mass Terms: Some Philosophical Problems. Synthese Language Library (Texts and Studies in Linguistics and Philosophy), vol 6. Springer, Dordrecht

Polzenhagen, F. (2007). Cultural conceptualisations in West African English: A cognitive-linguistic approach (Vol. 69). Peter Lang Pub Incorporated.

Qiang, Z (2014). The reflection of markedness in prototype category theory on semantic level and its implications for second language acquisition.

Riemer, N. (2010). Introducing semantics. Cambridge University Press.

Rosch, E. (1973). Natural categories. Cognitive Psychology, 4, 328-350.

Rosch, E. (1975). Cognitive representations of semantic categories. Journal of Experimental Psychology: General, 104, 192-233.

Rosch, E. (1975). Cognitive representations of semantic categories. Journal of experimental psychology: General, 104(3), 192. 
Rosch, E. (1977). Human Categorisation. In N. Warren (Ed.), Advances in Cross-cultural Psychology, Vol. 7. London: Academic Press.

Rosch, E., \& Mervis, C. B. (1975). Family resemblances. Cognitive Psychology, 7, 573-605.

Santos, D. (1990, August). Lexical gaps and idioms in Machine Translation. In Proceedings of the 13th conference on Computational linguistics-Volume 2 (pp. 330-335). Association for Computational Linguistics.

Schmitt, N. (2000). Vocabulary in language teaching. Ernst Klett Sprachen.

SOKOLOWSKI, R. (2017). Nouns, Verbs, and Contexts. In Presence and Absence: A Philosophical Investigation of Language and Being (pp. 12-22). WASHINGTON, D.C.: Catholic University of America Press.

Swarts, J. (2015). CONTEXT. In HEILKER P. \& VANDENBERG P. (Eds.), Keywords in Writing Studies (pp. 42-46). University Press of Colorado.

Takatori, Y., \& Schwanenflugel, P. (1992). Superordinate Category Terms and Mass-Count Noun Status. Journal of Linguistic Anthropology, 2(2), 199-209.

Taylor, R.J.(2015). Prototype Theory ion Linguistics. International Encyclopedia of the Social \& Behavioral Sciences (Second Edition),P. 286-289.

Weseliński, A. (1998). Papers on literature, culture and language. Warszawa: Wydawn. Uniw. Warszawskiego.

Zhang, F. (2011). Prototype Theory and the Categorization of the English Tense System. Linguistics Journal, 5(1).

Zhang, H. (2017). A study on implication of prototype theory in English Vocabulary teaching. Journal of Language Teaching and Research, 8(1), 133-137. 


\title{
APPENDIXES
}

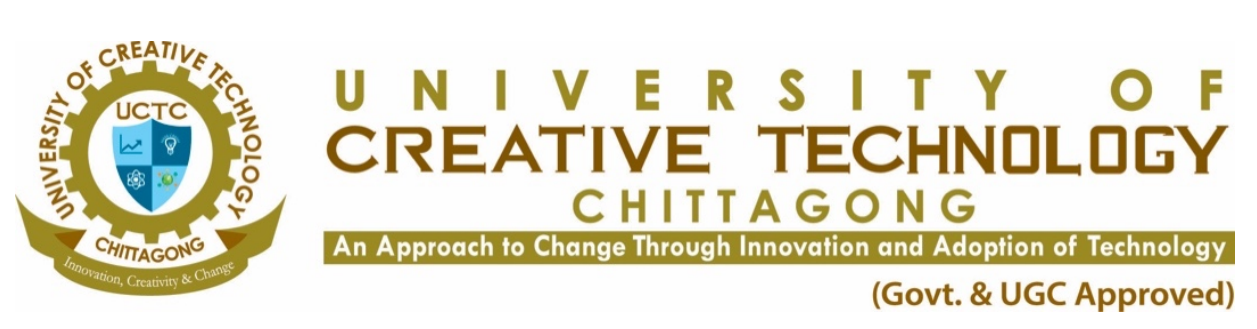

\author{
Faculty of Arts and Social Sciences \\ Department of English Language and Literature
}

\begin{abstract}
Mass Noun Decision (Prototyping) Task
This task is designed as a tool for collecting data for my research titled "Prototyping Mass Nouns: A Pedagogical Implication." I would sincerely appreciate your attempt to do the task which is expected not to take you more than 15 minutes.
\end{abstract}

Thank you very much.

Md. Saiful Alam (Saif)

Lecturer in English

Department of English Language and Literature (ELL)

Task 1: Look at the following list of nouns. Write features of mass nouns in the column B and order the nouns from the list according to their prototypicality in column A.

\section{Instructions:}

Put those examples which are typical members of mass noun in column 1.

Put those examples which are less typical members in Column 2.

Put those examples which are least typical members in Column 3.

Put those examples which are atypical in the column 4.

\section{List of nouns:}

wheat, time, gold, silver, , meat, flesh, childhood, sleep, stress, anger, height, happiness, health, , candy, toast, merchandise, hydrogen, nitrogen, advice, software, diamond, ice, heat, light, steel, coal, weather, grass, work, news, machinery, garlic, snow, hair sweet, scenery, astronomy, mathematics, shopping, rain, fuel, juice, wood, advertising, , anticipating, cotton oxygen, air, smoke, heat English Latin, Sanskrit, economics, biology, history, rubbish hockey, football, chess, checker, athletics, pneumonia, food, paper, mud, diabetes, safety, peace, knowledge, statistics, physics, transport, clothing, employment, adolescence, satisfaction research, logic, poetry, hope, furniture, soap, money, wealth, soccer, tennis, basketball, measles, polio, homework, cement, sugar, chalk, ice cream, Spanish, French, Chinese, traffic, equipment, mail, cash, bread, baggage influenza, water, milk, butter, oil, blood, coffee, tea, iron, sand, fire, , malaria, , music, beauty, friendship, education, grammar, success, salt, diamond, wine, honey, rice, sugar listening, swimming, running sunshine hypothyroidism, arthritis, , information, advice. 


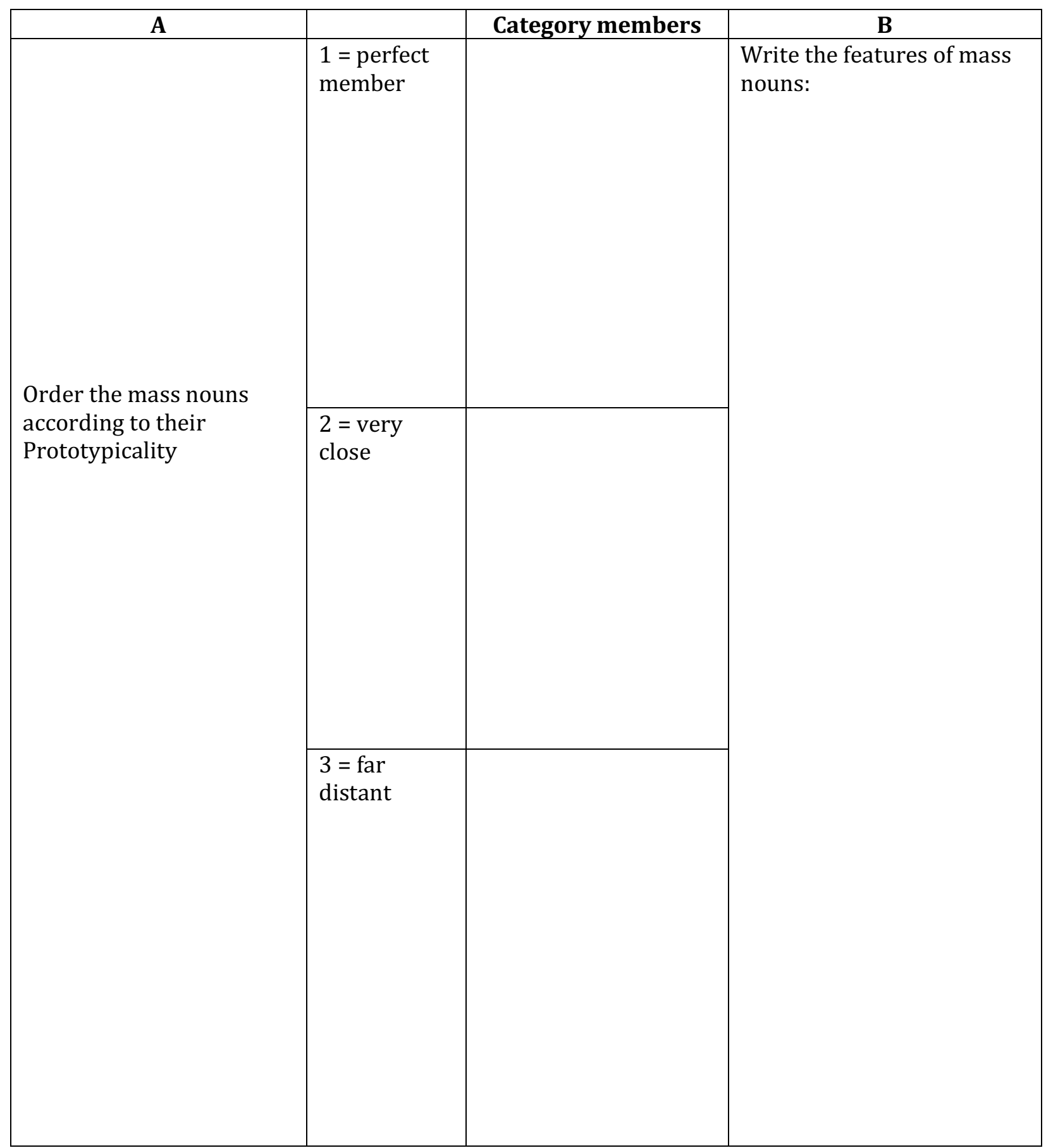

Now very quickly, would you mind filling in the following gaps with information about yourself?

Name:

Department:

Semester:

Age:

Years of English learning:

Have you taken / Are you taking any English language courses at university? If any, how many and which?

Which medium did you do your SSC and HSC in? __ Bangla / English.

Date: 